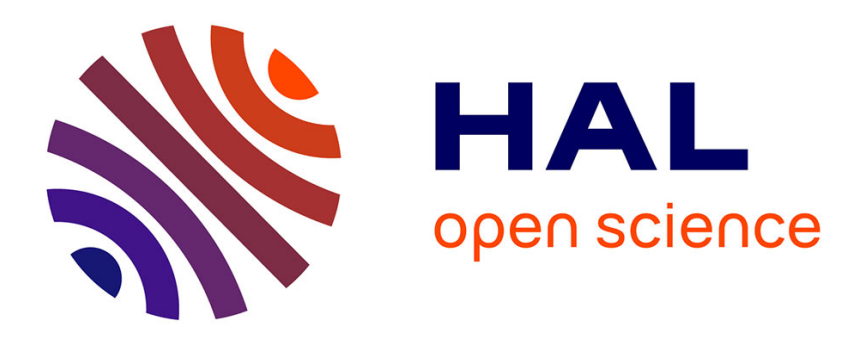

\title{
Poésie et politique dans l'Angleterre de la fin du Moyen Âge: le cas du Parlement
}

Aude Mairey

\section{To cite this version:}

Aude Mairey. Poésie et politique dans l'Angleterre de la fin du Moyen Âge: le cas du Parlement. Revue Française d'Histoire des Idées Politiques, 2007, 26, p. 231-250. hal-00239486

\section{HAL Id: hal-00239486 https://hal.science/hal-00239486}

Submitted on 8 Jan 2013

HAL is a multi-disciplinary open access archive for the deposit and dissemination of scientific research documents, whether they are published or not. The documents may come from teaching and research institutions in France or abroad, or from public or private research centers.
L'archive ouverte pluridisciplinaire HAL, est destinée au dépôt et à la diffusion de documents scientifiques de niveau recherche, publiés ou non, émanant des établissements d'enseignement et de recherche français ou étrangers, des laboratoires publics ou privés. 


\section{Poésie et politique dans l'Angleterre de la fin du Moyen Âge : le cas du Parlement}

L'affirmation du parlement anglais à la fin du Moyen Âge est un élément majeur de l'histoire politique de l'Angleterre, même s'il n'en est bien sûr pas le seul. Il s'est constitué, aux $\mathrm{XIII}^{\mathrm{e}}$ et $\mathrm{XIV}^{\mathrm{e}}$ siècles, dans les luttes séculaires entre les rois et les grands barons pour le partage - ou non - du pouvoir, ainsi que dans la tourmente de la Guerre de Cent ans. À l'origine de cette institution se trouve pour une grande part la question de la fiscalité, que le gouvernement royal entreprend d'élargir du fait, surtout, de ses dépenses liées à la guerre. C'est le principe de l'acceptation d'une fiscalité directe, selon le fameux principe de droit romain Quod omnes tangit ab omnibus tractari et approbari debet, qui a présidé à la formation du parlement, alors que celui-ci n'était encore, au XIII ${ }^{\mathrm{e}}$ siècle, qu'un Grand Conseil élargi. Au siècle suivant en revanche, les fonctions de cette assemblée se multiplient : c'est une haute cour de justice; on y présente les pétitions au roi dont certaines prennent rapidement un caractère général (les pétitions des Communes) et sont de plus en plus à l'origine de la législation par statut ; mais surtout, on y discute des subsides et des taxes ${ }^{1}$.

À partir du second quart du XIV ${ }^{\mathrm{e}}$ siècle, la composition du parlement est devenue plus ou moins stable ${ }^{2}$. Étaient convoqués les grands barons (les Lords laïcs et ecclésiastiques), mais aussi des représentants des Commons (les Communes), membres de la petite noblesse et représentants des villes ${ }^{3}$. Ces dernières catégories occupent alors une place croissante dans le fonctionnement de la monarchie et de l'État moderne ${ }^{4}$ : la gentry ${ }^{5}$ - petite noblesse rurale très impliquée dans l'administration locale (les chevaliers et les squires) - exerce notamment un grand nombre de charges par l'intermédiaire des offices et des commissions qui lui sont confiées ${ }^{6}$. Quant aux hommes de loi, dont beaucoup s'intègrent d'ailleurs à la gentry, ils deviennent de plus en plus indispensables au fur et à mesure du développement de la Common Law, le droit anglais ${ }^{7}$. Enfin, les marchands anglais évincent de plus en plus les marchands étrangers (surtout italiens) qui, auparavant, dominaient le commerce de l'île et, en particulier, celui de la laine. Ils jouent un rôle-clé dans la gestion municipale et dans celle des finances du pays, y compris sur le plan de la fiscalité. Ces différents groupes s'ajoutent au roi et à la noblesse au sens strict, les earls (comtes) et les lords, pour former la société politique au sens restreint du terme, c'est-à-dire ceux qui participent d'une façon plus ou moins directe à l'exercice du pouvoir.

$\mathrm{Au}$ parlement, ce sont surtout les Communes qui s'affirment, car c'est d'abord vers elles que le roi se tourne pour obtenir des fonds. Par là même, il a dû faire face aux exigences croissantes de ses membres. Ainsi une identité collective des Communes voit-elle peu à peu le jour et, à la fin du règne d'Édouard III, cette importance est concrétisée par l'apparition d'un

\footnotetext{
${ }^{1}$ Parmi une littérature abondante, voir notamment G. Harriss, King, Parliament and Public Finance in Medieval England to 1369, Oxford, 1975 ; The English Parliament in the Middle Ages, éd. R. G. Davies et J. H. Denton, Philadelphie, 1981 et en français, J.-P. Genet, Genèse de l'État moderne, Culture et société politique en Angleterre, Paris, 2003.

${ }^{2}$ Le parlement de 1322 est à cet égard considéré comme le parlement "modèle".

${ }^{3}$ Les clercs n'étant pas représentés en tant qu'ordre, le Parlement n'est pas une assemblée des États.

${ }^{4}$ Sur cette notion, voir J.-P. Genet, "Genèse de l'Etat moderne : les enjeux d'un programme de recherche", Actes de la recherche en sciences sociales 118, 1997, p. 3-18.

5 Cf. F. Lachaud, "La 'formation de la gentry', fin XII ${ }^{\mathrm{e}}$-mi $\mathrm{XIV}^{\mathrm{e}}$ siècles: un nouveau concept historiographique ?", dans Histoires d'Outre-Manche : Tendances récentes de l'historiographie britannique, éd. F. Lachaud, I. Lescent-Giles, F. Ruggiu, Paris, 2001, p. 13-36.

${ }^{6}$ Les offices étaient des charges ayant une durée déterminée (parfois à vie), alors que les commissions avaient un caractère ponctuel et temporaires (commissions d'enquête...).

${ }^{7}$ Sur la formation et l'importance de la Common Law en Angleterre, voir notamment Paul Brand, The Making of the Common Law, Londres, 1992.
} 
Speaker, sorte de porte-parole des Communes ${ }^{8}$. Il faut cependant souligner que ces dernières, malgré leurs exigences et leurs critiques - surtout lors des périodes de crise - ne réclament pas le gouvernement du royaume qui est la prérogative du roi (et des grands barons). Elles veulent surtout que l'on tienne compte de leur conseil, ce qui est néanmoins un élément essentiel, car le conseil est à la fois un droit et un devoir ${ }^{9}$. Le Parlement est donc avant tout un espace de dialogue, un lieu d'expression de la société politique anglaise, ou du moins de ses plus éminents représentants ${ }^{10}$. Mais ce dialogue est-il si aisé ?

$* * * * * * *$

Les sources qui permettent d'appréhender l'histoire du parlement sont d'abord des sources normatives. Parmi elles, les plus importantes sont les Rotuli Parliamentorum, les rouleaux du parlement, comptes-rendus des clercs du parlement, de plus en plus fournis à partir du milieu $\mathrm{du} \mathrm{XIV}^{\mathrm{e}}$ siècle $^{11}$. Il faut noter aussi l'existence d'un texte intéressant mais difficile à interpréter, le Modus Tenendi Parliamentum, qui décrit la composition et les procédures du parlement ${ }^{12}$. Il y a également un certain nombre de sources éparses, comme les writs de convocations, qui ont été utilisées pour construire une étude prosopographique poussée des membres du parlement, fort précieuse ${ }^{13}$. Par ailleurs, les chroniques évoquent parfois les parlements, en s'attardant généralement sur les moments de crise, souvent cruciaux pour la construction des rapports entre les différentes composantes de la société politique ${ }^{14}$. Mais ces sources, si intéressantes soient-elles (encore qu'elles laissent de nombreuses zones d'ombres, en particulier sur le déroulement des débats) peuvent être complétées par les sources littéraires. Ces dernières sont fort riches en Angleterre dans les derniers siècles du Moyen Âge, en particulier les sources en anglais. Cela est lié à plusieurs facteurs, et d'abord à l'extension de la literacy - l'aptitude à lire et à écrire - à des groupes de plus en plus nombreux, surtout laïcs (les clercs n'ont alors plus le monopole de la culture). En Angleterre, cette extension a été particulièrement nette à partir du XIII ${ }^{\mathrm{e}}$ siècle, et surtout au XIV ${ }^{\mathrm{e}}$ siècle. À la fin du Moyen Âge, l'Angleterre est un véritable "creuset culturel", et les sources littéraires sont un élément important pour comprendre l'évolution de la société de ce pays ${ }^{15}$.

\footnotetext{
${ }^{8}$ Le premier Speaker est Peter de la Mare, en 1376. Sur les Speakers, voir J. S. Roskell, The Commons and their Speakers in English Parliament, 1376-1523, Manchester, 1965.

${ }^{9}$ Sur la notion de conseil, voir J.-P. Genet, "De Richard II à Richard III : le conseil", dans «A l'ombre du pouvoir ». Les entourages princiers au Moyen Âge, éd. A. Marchandisse et J. -L. Kupper, Genève, 2003, p. 177202, mais aussi J. Watts, "The Counsels of King Henry VI, c. 1435-1445”, English Historical Review 106, 1991, p. 279-298 et J. Ferster, Fictions of Advice : The Literature and Politics of Counsel in Late Medieval England, Philadelphie, 1996.

${ }^{10}$ Sur cette question, voir Genet, Genèse de l'Etat moderne, op. cit., p. 89 et suivantes.

${ }^{11}$ Rotuli Parliamentorum; ut et petitiones et placita in Parliamento tempore Édouardi R. I (Édouardi II, Édouardi III, Ricardi II, Henrici IV, V, VI, Édouardi IV, Ricardi III, Henrici VII), 1278-1503, éd. J. Strachey et alii, 6 vol., Londres, 1767-77. Cf. J. Taylor, English Historical Literature in the Fourteenth Century, Oxford, 1987, p. 196 : "After 1339 parliamentary records in the form of the rolls assume the character which they were to retain for the remainder of the century, namely a record of the business of parliament, particularly of the pleas and jugdments of the court of parliament. They were not a record of debates, nor did they give the chronological order in which matters came before that assembly".

${ }^{12}$ Le texte est édité dans Parliamentary Texts of the Middle Ages, éd. N. Pronay et J. Taylor, Oxford, 1980. Les controverses sur sa date comme sur sa nature sont grandes. Pour certains, c'est un traité "administratif" datant de la première moitié du XIV siècle, pour d'autres un texte politique de la fin de ce même siècle. Les arguments en faveur de la première hypothèse sont cependant plus généralement soutenus.

${ }^{13}$ The History of Parliament, the House of Commons, 1386-1421, éd. J. S. Roskell, 4 vol., Stroud, 1992.

${ }^{14}$ Cf. Taylor, English Historical Literature, op. cit., p. 195-216.

${ }^{15}$ Sur ces questions, voir notamment, Genet, Genèse de l'État moderne, op. cit., passim ; M. Clanchy, From Memory to Written Record: England 1066-1307, Londres, 1979¹, 1992 ; J. Coleman, Medieval Readers and
} 
Quel peut être l'apport de ces sources pour l'historien, dans le cas particulier du parlement ${ }^{16}$ ? Elles contribuent, selon nous, à appréhender la perception qu'avaient de cette institution certains contemporains, plus ou moins proches de la société politique à laquelle nous faisions allusion plus haut et parfois même proches du milieu parlementaire. Elles permettent aussi de s'interroger sur la nature de cette institution et de la replacer dans le cadre plus général d'une réflexion sur l'instauration d'un dialogue entre pouvoir et société politique, au cœur de la naissance de l'État moderne. Mais cette instauration ne va pas sans tensions et dans de nombreuses œuvres littéraires, ces dernières sont exprimées sous la forme d'un fort questionnement sur la validité des différents discours, et notamment du discours de conseil : qui peut (et doit) donner son conseil, au nom de quoi et pourquoi. C'est dans ce cadre que certains auteurs se sont interrogés sur le parlement et leurs perceptions constituent un élément précieux pour comprendre comment s'est construit le dialogue entre pouvoir et société politique que nous évoquions et quelle y a été la place du parlement.

$* * * * * * *$

Les textes littéraires qui évoquent le parlement d'une manière ou d'une autre sont assez peu nombreux et ceux qui le font en sont d'autant plus intéressants. Nous en retiendrons quatre, qui datent tous de la fin du $\mathrm{XIV}^{\mathrm{e}}$ et du début du $\mathrm{XV}^{\mathrm{e}}$ siècle, grand moment d'affirmation du parlement, marqué notamment par deux crises importantes, celle de la fin du règne d'Édouard III qui a vu s'affirmer l'unité des Communes au "Bon Parlement" (Good Parliament) de 1376 avec l'apparition de leur Speaker, et surtout celle de la déposition de Richard II et de son remplacement par Henry IV de Lancastre en $1399^{17}$. Ces poèmes, tous en anglais, sont The Parliament of Fowles de Geoffrey Chaucer, Piers Plowman de William Langland, Richard the Redeless et Mum and the Sothsegger. Ils font presque tous appel au rêve, qui était alors très à la mode dans la littérature de l'époque ${ }^{18}$. Le contraste entre le poème de Chaucer et les trois autres textes est au premier abord assez net. Les auteurs de ces derniers évoquent plus ou moins directement le contexte contemporain et se livrent à une critique sociale et politique importante (mais néanmoins réformatrice) ${ }^{19}$, alors que The Parliament of Fowles est (apparemment) d'abord un poème sur l'amour et que le parlement qui y est mis en scène - celui des oiseaux - débat de cette question. Pourtant, malgré ces différences, il existe des interrogations communes, que nous allons nous attacher à définir.

Ces poèmes sont d'ailleurs issus de contextes assez différents. Geoffrey Chaucer (v. 13401400) est l'écrivain le plus important de la fin du Moyen Âge anglais. C'est un poète lié d'assez près à la cour du roi, même s'il n'existe pas en Angleterre, comme en France à la même époque, de véritable poète de $\operatorname{cour}^{20}$. Il est lié au duc de Lancastre, John of Gaunt

Writers. English Literature in History, 1350-1400, Londres, 1981 ; M. Parkes, "The literacy of the laity", dans Literature and Western Civilization: The Medieval World, éd. D. Daiches et A. K. Thorlby, Londres, 1973, p. 555-76.

${ }^{16}$ Pour une discussion générale sur l'intérêt des sources littéraires pour l'historien, nous nous permettons de renvoyer à l'introduction de notre ouvrage issu de notre thèse de doctorat, Une Angleterre entre rêve et réalité Littérature et société en Angleterre au XIV siècle, à paraître aux Publications de la Sorbonne.

${ }^{17}$ Sur la crise de 1376, voir G. Holmes, The Good Parliament, Londres, 1975 ; sur l'usurpation d'Henry de Lancastre, parmi une immense littérature, voir notamment N. Saul, Richard II, Yale, 1997 et Henry IV: The Establishment of the Regime, 1399-1406, éd. G. Dodd et D. Biggs, Woodbridge, 2003.

${ }^{18}$ Cf. A. Strubel, Grant senefiance a : Allégorie et littérature au Moyen Âge, Paris, 2002 ; A. Spearing, Medieval Dream-Poetry, Cambridge, 1976.

${ }^{19}$ Nous avons étudié ces trois textes de manière détaillée dans Une Angleterre entre rêve et réalité, op. cit.

${ }^{20}$ Sur cette question, voir R. F. Green, Poets and Princepleasers : Literature and the English Court in the Late Middle Ages, Toronto, 1980. 
(troisième fils du roi Édouard III et père du futur Henry IV) et il a effectué de nombreuses missions pour le gouvernement royal ${ }^{21}$. Mais surtout, Chaucer est sans doute le premier auteur - au sens moderne du terme - reconnu en Angleterre de son vivant (même s'il s'inscrit bien sûr dans un contexte beaucoup plus général ${ }^{22}$ ). Son œuvre, écrite d'abord en anglais, comprend à la fois des créations et des traductions et certains de ses textes ont connu une diffusion considérable. Du plus célèbre d'entre eux, un recueil en fait, les Canterbury Tales ${ }^{23}$, il nous reste plus de 80 manuscrits et son audience a été diversifiée sur le plan social ${ }^{24}$. Son impact a été absolument considérable et sans équivalent en France : il a très vite été reconnu et façonné comme une figure fondatrice de la littérature anglaise par ses successeurs immédiats (encore aujourd'hui, certains Anglais le comparent volontiers à Dante, même si d'autres commencent à réfléchir sur le mythe chaucérien) ${ }^{25}$. The Parliament of Fowles, le texte qui nous intéresse ici, a été écrit durant la période 1373-1385, mais il est difficile d'être plus précis ${ }^{26}$. Il nous est conservé dans 14 manuscrits ${ }^{27}$ et a fait l'objet de très nombreuses interprétations portant en particulier sur les réflexions de Chaucer sur l'amour, la nature..., qui ne nous retiendront pas $\mathrm{ici}^{28}$. Le narrateur s'endort alors qu'il lisait un livre, le Commentaire sur le songe de Scipion de Macrobe (auteur de la fin de l'Antiquité) ; Cicéron raconte en effet ce songe dans la deuxième partie de son De Re Publica ${ }^{29}$. Il se met alors à rêver de Scipion l'Ancien qui le conduit dans un jardin merveilleux au milieu duquel se trouve un temple, dans lequel il croise de nombreuses allégories (Amour, Plaisir, Courtoisie, Noblesse, Paix, mais aussi Jalousie, Priapus...). Dans le temple se trouvent Vénus et Richesse, ainsi que de nombreuses fresques décrivant des histoires d'amour. En sortant, il voit la déesse Nature qui s'apprête à recevoir les oiseaux en audience. C'est le jour de la SaintValentin et ils doivent choisir leur compagne. S'ensuit une description des oiseaux selon leurs états (vers 323-364) : les oiseaux de proie, les oiseaux à graine, les oiseaux à vers et les oiseaux d'eaux. Les érudits ont proposé divers parallèles pour ces états. Selon Paul Olson, ils correspondraient dans l'ordre aux nobles, aux clercs, aux gens du commun (les marchands surtout) et aux administrateurs ${ }^{30}$. Quoi qu'il en soit, Nature les presse de choisir leur compagne, mais un conflit éclate entre les oiseaux de proie, les plus nobles, à propos d'une belle aigle qui se trouve sur la main de Nature. C'est alors que les discussions entre oiseaux à propos de ce conflit se transforment en véritable parlement. Finalement, Nature tranche le

\footnotetext{
${ }^{21}$ Sur la vie de Chaucer, voir D. Pearsall, The Life of Geoffrey Chaucer, Oxford, 1992. L'ensemble de ses œuvres (reconnues) est édité dans le recueil The Riverside Chaucer, dir. L. Benson, Oxford, 1987. C'est l'édition que nous utilisons.

${ }^{22}$ Sur l'évolution de la notion d'auteur à la fin du Moyen Âge, voir J.-P. Genet, La mutation de l'éducation et de la culture médiévales, 2 vol., Paris, 1999, vol. 2, p. 405-408.

${ }^{23}$ Les Contes de Canterbury ont été traduits intégralement en français par André Crépin (Paris, 2001).

${ }^{24} \mathrm{C}$ 'est le plus grand nombre de manuscrits restant pour une œuvre littéraire anglaise à la fin du Moyen Âge. Cf. P. Strohm, "Chaucer's Fifteenth Century Audience and the Narrowing of the "Chaucer Tradition"', Studies in the Age of Chaucer 4, 1982, p. 3-32; "Chaucer's audience(s) : Fictional, Implied, Intended, Actual", Chaucer Review 18, 1983, p. 137-64 ; Social Chaucer, Cambridge, Mass., 1989.

${ }^{25}$ Voir notamment N. Watson, "The Politics of Middle English Writings", dans The Idea of Vernacular, An Anthology of Middle English Literary Theory, 1280-1520, éd. J. Wogan-Browne, N. Watson, A. Taylor, R. Evans, Exeter, 1999, p. 331-352.

${ }^{26}$ En fait, le texte est sans doute postérieur à 1376, dans la mesure où Chaucer évoque les Speakers en Parlement, qui n'apparaissent qu'à cette date.

${ }^{27}$ Cf. M. C. Seymour, "The manuscripts of Chaucer's Parliament of Fowles", Scriptorium 47, 1993, p. 192-204.

${ }^{28}$ Pour une vue d'ensemble, cf. A. Minnis, The Shorter Poems, Oxford Guides to Chaucer, Oxford, 1995.

${ }^{29}$ Macrobe, Commentaire sur le Songe de Scipion, éd. et trad. M. Armisen-Marchetti, Paris, 2001. Cet ouvrage a eu une influence importante sur la conception du rêve au Moyen Âge.

${ }^{30}$ P. Olson, "The Parliement of Fowles : Aristotle's Politics and the Foundation of Human Society", Studies in the Age of Chaucer 2, 1980, p. 53-69, p. 61-63.
} 
débat en décidant que le choix reviendra à la belle, qui demande un délai. Les oiseaux choisissent alors leurs compagnes et se séparent en chantant.

William Langland (v. 1330-v. 1380) est un autre auteur majeur du XIV ${ }^{\mathrm{e}}$ siècle anglais. Il nous est beaucoup moins bien connu que Chaucer ${ }^{31}$. C'était sans doute un clerc n'ayant reçu que les ordres mineurs qui a travaillé pour des laïcs ; il a peut-être également été scribe. En tout cas, il n'est pas du tout lié à la cour comme Chaucer, même s'il est peu vraisemblable qu'il n'ait pas eu de connexions bien placées, étant donné la rapidité de la diffusion de son œuvre. Son poème, Piers Plowman, est l'œuvre d'une vie : il nous en reste plusieurs versions composées entre le début des années 1360 et la fin des années $1380^{32}$. Son succès a été grand : les différentes versions nous sont conservées dans plus de 50 manuscrits. La version $\mathrm{B}$, que nous évoquerons ici et qui comprend plus de 7000 vers, est structurée en vingt passus (chapitres) et huit visions différentes séparées par les réveils et les assoupissements successifs du rêveur. Les deux premières visions sont centrées sur la société humaine (prologue et passus 1 à 7). Les troisièmes et quatrièmes visions (passus 8 à 14) nous représentent le narrateur, Will, en quête d'un concept volontairement flou, Bien-Faire (Dowel). Durant sa recherche, il rencontre de nombreuses figures d'autorité et explore différents modes cognitifs et psychologiques. Dans les dernières visions, enfin, le poète se tourne, après une critique nourrie de l'institution ecclésiastique, vers la vie et la passion du Christ et la naissance allégorique de l'Église, la forteresse Unité. Le poème se termine cependant sur une note pessimiste, puisque cette forteresse est attaquée par l'Antéchrist et son armée et que Conscience, un des principaux personnages du poème, s'en va à la recherche de Piers Plowman, figure messianique, et de Grace. Dans les pages qui suivent, nous nous concentrerons surtout sur la première vision.

Sur les deux derniers poèmes retenus, Richard the Redeless et Mum and the Sothsegger, les moins diffusés (il ne nous reste qu'une copie pour chacun et pour les deux, la fin manque), nous savons peu de choses ${ }^{33}$. Ils ont été composés au début du $\mathrm{XV}^{\mathrm{e}}$ siècle, peut-être par le même auteur (pendant fort longtemps, les érudits anglais ont d'ailleurs pensé que c'étaient deux parties d'un même poème). Richard a été écrit juste après la déposition de Richard II. L'auteur s'adresse au roi déchu et lui explique pourquoi il est dans ce triste état (au moment où l'auteur écrit, Richard est d'ailleurs déjà mort). C'est en fait une manière de s'adresser au nouveau pouvoir, pour que ce dernier ne renouvelle pas les erreurs de l'ancien. Et dans le dernier des quatre passus, il esquisse une violente satire du parlement (malheureusement inachevée). Dans Mum, le narrateur part en quête d'un sothsegger, un diseur de vérité, et passe en revue toutes les catégories de la société politique active : les administrateurs, le clergé, les nobles, les élites urbaines. Chaque fois, il est confronté à la flatterie et à la corruption, par le biais du personnage de Mum, incarnation de ces vices qui sont bien pour le narrateur des vices politiques et sociaux, s'opposant au bon fonctionnement du gouvernement. Dépité par ses rencontres, rabroué par Mum, il s'endort, se met alors à rêver et rencontre un apiculteur qui lui explique ce qu'est réellement un diseur de vérité et surtout où il se trouve : dans le cœur de l'homme. Il conseille alors au narrateur de faire un livre pour transmettre la vérité. La fin du poème - inachevé - est une critique renouvelée des différentes catégories envisagées précédemment. Richard the Redeless et Mum and the Sothsegger sont les deux poèmes qui s'expriment le plus directement sur le parlement, et ce dans un contexte marqué

\footnotetext{
${ }^{31}$ Voir notamment R. Hanna, William Langland, Aldershot, 1993 ; K. Kerby-Fulton, «Piers Plowman », dans The Cambridge History of Medieval English Literature, éd. D. Wallace, Cambridge, 1999, p. 513-538.

${ }^{32}$ Les éditions de Piers Plowman sont extrêmement nombreuses. Les citations données proviennent de l'édition d'A.V.C. Schmidt (Londres, $1987^{2}$ ). Nous renvoyons le lecteur à notre traduction, parue aux Publications de la Sorbonne en 1999.

${ }^{33}$ Ces poèmes ont été édités par Helen Barr: The Piers Plowman tradition, Londres, 1993. Voir aussi son ouvrage, Signs and Soth : language in the Piers Plowman tradition, Oxford, 1994.
} 
par ce que Paul Strohm a appelé "l'anxiété de la légitimation", liée à l'usurpation de la couronne par Henry de Lancastre en $1399^{34}$.

Pour comprendre les perceptions qu'avaient les poètes du parlement, nous présenterons tout d'abord les éléments de description de cette institution dans les poèmes, avant de nous pencher sur les critiques plus ou moins voilées des membres du parlement, qui semblent parfois contradictoires. Enfin, nous tenterons de replacer ces critiques dans le cadre de la réflexion plus générale des poètes sur la validité du discours, et notamment du discours de conseil.

$* * * * * * *$

Tout d'abord, nos poètes savent visiblement de quoi ils parlent lorsqu'ils évoquent le parlement. La description la plus précise apparaît dans Richard the Redeless, par exemple lorsque l'auteur évoque les mécanismes de convocation :

Ils inventèrent quelque folie qui ne leur manquait jamais, et décidèrent par ruse à leur conseil du matin, de tenir un parlement secret pour leur profit. Ils firent écrire des writs scellés à la cire, pour les pairs et les prélats qui devaient venir, et ils envoyèrent là-dessus des mandats aux shérifs, pour qu'ils choisissent des chevaliers comme le mandat le voulait, pour représenter le comté en compagnie des grands.

Et quand vint le jour de l'action, que les seigneurs et les chevaliers du comté furent assemblés, alors, selon la procédure initiale, on commença à déclarer la cause de leur venue et ensuite la volonté du roi. De manière appropriée, un clerc commença par ces mots, et prononça les points [du discours] ouvertement pour tous ; il discouru pour [demander de] l'argent plus que pour tout autre chose, en flattant les grands pour que les réclamations ${ }^{35}$ ne puissent s'exprimer.

Richard the Redeless IV, vers 23-38

La procédure décrite dans ces vers est en grande partie conforme à ce que l'on en connaît par le biais des sources normatives : convocations envoyées individuellement aux lords, mandats envoyés aux shérifs (représentants du roi dans les comtés), discours d'ouverture du parlement, généralement prononcé par le chancelier qui était presque toujours un clerc, pour exposer les motifs de la convocation. Cependant, la critique est déjà présente : les shérifs, par exemple, n'étaient pas censés choisir les représentants mais organiser des élections en convoquant la cour du comté. C'était d'ailleurs une critique commune, que l'on retrouve souvent dans les pétitions parlementaires.

Dans les autres textes, cette connaissance est plus diffuse, mais bien présente. Chaucer, par exemple, semble bien informé des procédures d'élection des Speakers :

Nature, qui prêtait toujours une oreille au murmure de fond de l'ignorance, dit d'une voix éloquente : "Tenez vos langues là-bas ! Et je trouverai bientôt, je l'espère, un conseil pour me libérer de ce bruit; je juge que de chaque race, un devrait être appelé, pour prononcer le verdict pour vous tous".

Cette décision fut approuvée par tous les oiseaux ; et les oiseaux de proie choisirent d'abord, par une élection ouverte, le jeune faucon pour résumer l'essentiel, et arriver à une conclusion, comme il lui plairait. Et il alla se présenter à Nature, qui l'accepta volontiers.

Parliament of Fowles, vers 519-532

Il y a cependant dans le poème une différence notable d'avec le parlement "réel" : dans ce dernier, il n'y a qu'un seul Speaker (pour les Communes), alors que dans le texte de Chaucer, un porte-parole est nommé par catégorie d'oiseaux : le faucon pour les oiseaux de proie, la

\footnotetext{
${ }^{34}$ P. Strohm, England's Empty Throne : Usurpation and the language of legitimation, 1399-1422, New Haven, 1998.

${ }^{35}$ Les greuyes (grievances en anglais moderne) étaient les revendications effectuées par les Communes en parlement.
} 
tourterelle pour les oiseaux à graine, le canard pour les oiseaux d'eau et le coucou pour les oiseaux à vers.

On le voit donc, les poètes font preuve d'une bonne connaissance des procédures parlementaires $^{36}$. L'éditrice de Richard pense même que l'auteur de ce texte avait probablement assisté à un parlement. La réflexion présente dans les poèmes est celle de gens bien informés.

$* * * * * * *$

Mais ces gens bien informés ne se privent pas de critiquer le parlement ou plutôt ses membres, même si, et peut-être surtout parce que, c'est pour eux une institution essentielle. Dans Richard et dans Mum tout d'abord, le parlement semble l'institution la plus apte à former une courroie de transmission entre le roi et ses sujets, plus, en tout cas, que le conseil royal restreint, très critiqué dans les deux poèmes. Il est pourtant suggéré à plusieurs reprises que cette responsabilité du parlement fait défaut et ce d'abord parce que les Lords et les représentants des comtés et des villes n'accomplissement pas leur devoir :

Alors certains étaient assis comme le zéro de l'arithmétique, qui prend une place mais ne contribue à rien ; et certains avaient dîné la veille avec Simonie, ils représentèrent le comté, mais n'y gagnèrent rien ; certains étaient tituleris et allèrent vers le roi, et l'informèrent sur des ennemis qui semblaient de bons amis, qui parlaient pour le mieux et ne cherchaient aucun blâme du roi, du conseil ou des communes, si quelqu'un faisait attention à la signification ; certains s'assoupissaient, dormaient et ne disaient rien ; certains marmonnaient avec la bouche et ne savaient pas ce qu'ils disaient ; certains avaient une pension ${ }^{37}$ et y adhéraient à jamais et ne voulaient pas s'avancer par peur de leurs maîtres ; certains étaient si limités et engourdis dans leurs esprits, qu'ils étaient dans la confusion s'ils venaient à la conclusion ; ainsi aucun homme de loi, de bourgeois ou autre ne pouvait interpréter les conclusions, si aveugle, plate et nue était la raison.

Et certains étaient si agressifs à la première occasion, qu'ils déployaient une voile de plus et portaient le hunier devant le vent fraîchement pour faire un bon voyage. Alors les seigneurs se tenaient sous le vent avec leur lourde cargaison et avec les charges, et ils faisaient virer de bord le bateau et blâmaient le maître, qui ne connaissait pas le cours naturel qui appartient au métier, et l'avertissaient sagement du côté au vent. Alors le mât du milieu, à la fin du mois, se courba à en rompre et les jeta à terre ; s'ils n'avaient pas amené un ris et barré le bateau au mieux et amené une voile avant la venue de la tempête, ils auraient tous été jetés par-dessus bord. Certains étaient déjà troublés par le conseil, et savaient bien comment cela se terminerait, avant que certains de l'assemblée ne se repentent. Certains se tenaient avec la majorité quoi qu'il arrive, et certains se tenaient droits et n'iraient pas plus avant. Certains parlaient ouvertement comme il fut prouvé après, et réclamaient davantage l'argent que le roi leur devait que le bien des communes qui payaient leurs dépenses, et on leur promettait la récompense d'être compensés assurément du même argent, s'ils désiraient aider. Et certains craignaient les ducs, et délaissaient Bien-Faire...

Richard the Redeless IV, vers 53-93

Ce passage, non dénué d'humour, résume tous les défauts de ceux qui siègent en parlement : la lâcheté, mais aussi la corruption et, sur un plan plus général, la défense des intérêts particuliers au détriment d'un intérêt commun. L'image du bateau, courante mais habituellement utilisée pour figurer l'Angleterre (et dans un sens positif), le souligne avec force. Ces vers ont été considérés comme une satire du parlement tenu à Shrewsbury en 1398, au cours duquel Richard II consomma son (éphémère) triomphe sur ses opposants, les Appellants $^{38}$ et obtint un très généreux subside de la part des Commons ${ }^{39}$. Il a cependant, nous le verrons, une portée plus générale que celle de la simple critique conjoncturelle.

\footnotetext{
${ }^{36}$ Il faut noter que le fils de Chaucer, Thomas Chaucer fut un pilier assidu du parlement dans les premières décennies du $\mathrm{XV}^{\mathrm{e}}$ siècle : il fut 5 fois Speaker, ce qui est un record absolu pour toute la période médiévale.

${ }^{37}$ Le terme hire en anglais peut à la fois faire référence à la pension versée par les seigneurs à ceux qu'il retenaient à leur service (les retainers), et à la somme versée aux chevaliers du comté pour couvrir leurs frais.

${ }^{38}$ En 1386-1388, les Lords Appellants - Thomas de Woodstock, duc de Gloucester, Richard Fitzalan, earl d'Arundel, Thomas Arundel, évêque d'Ely, rejoints en 1387 par Thomas Beauchamp, earl de Warwick et par
} 
Dans Mum, le narrateur rappelle aux représentants du parlement les dangers de la fréquentation de Mum, qui constitue dans le poème l'incarnation par excellence de la perversion du discours :

De tous les maux et les mauvais gouvernements qui croissent dans le royaume, Mum a été l'instigateur toutes ces années et aussi la racine et le modèle, je peux bien l'avouer. Je pense prouver cela d'abord par le parlement : quand les chevaliers sont venus pour cette action pour la communauté et se sont assemblés, [ce devait être] même s'ils devaient en mourir - pour montrer les malheurs du royaume, et ne ménager aucun discours, pour faire éclater les furoncles et les boursouflures et laisser la colère sortir en courant, sous peine que le faux félon n'éclate à l'intérieur. Car comme je l'ai entendu, on soigne bien plus tôt quand la colère et le poison sont drainés au-dehors : il est mieux de [les] faire éclater quand le remède peut s'appliquer que de se soulever contre les regalia et troubler le royaume.

L'absence de cette vertu provoque la croissance du venin et des blessures sans remèdes dans de nombreux lieux divers, [et ce] depuis que les seigneurs et les hommes des comtés évitent la vérité dans les lieux où il est approprié de parler pour le royaume, de converser des problèmes et de s'efforcer de les réparer. Car tous les points dangereux des prélats et d'autres, tels que les pairs qui ont le pouvoir de piller et de faire des dons, ils ne les évoqueront pas à cause du danger qui pourrait survenir, mais cacheront toutes les lourdeurs et hésiteront chacun ; ils feront de Mum leur messager pour qu'il détermine leur procès et ramèneront à la maison un sac plein de furoncles non soignés dont la nécessité est par nature de les ennuyer ensuite.

Mum and the Sothsegger, vers 1115-1140

Ce passage a une construction difficile mais le sens en est clair. À cause de Mum, les représentants des Communes et les Lords ne s'expriment pas au parlement. Or, seule la connaissance des problèmes peut permettre de soulager le royaume. Nous voyons l'importance accordée à l'institution en tant que lieu de discussion, qui renforce cette impression de la nécessité d'une saine réaction face à Mum. Cela est appuyé par la présence soutenue d'images empruntées au registre médical.

Dans les deux poèmes que nous venons d'envisager, les membres du parlement sont avant tout critiqués parce qu'ils ne parlent pas, alors qu'ils ont l'autorité pour le faire. Dans les textes de Langland et Chaucer, la réflexion est plus complexe et plus ambiguë.

Dans le poème de Langland, le parlement apparaît dans la première vision, qu'il nous faut évoquer rapidement. Dans le prologue, le narrateur s'endort et se retrouve dans un champ où se trouvent divers membres de la société, qu'il décrit. Il assiste également à une fondation idéale de la royauté et c'est à ce moment qu'il évoque le parlement par le biais d'une fable. Au passus I, Holy Chirche (Sainte Église) apparaît et entreprend d'expliquer au narrateur la signification de sa vision. Elle fait ensuite un long sermon, centré sur les notions de Truthe et de Love. Au passus II, Le narrateur demande comment reconnaître le vrai du faux. Holy Chirche lui répond en dénonçant $M e d e^{40}$, puis disparait. Il assiste alors au mariage de Mede et de Fals, ce qui est l'occasion de nouvelles critiques sociales. L'affaire est portée devant le roi par Conscience, mais seule Mede se retrouve à Westminster. Dans le passus suivant, nombreux sont ceux qui offrent leur soutien à Mede: des juges, des clercs, un frère qui la confesse et des maires. Le roi la sermonne et lui propose d'épouser Conscience, mais celui-ci refuse. S'ensuit alors un débat entre lui et Mede qui se termine par la défaite de cette dernière.

\footnotetext{
Henry Bolingbroke, duc de Lancastre (futur Henry IV) - s'opposèrent à Richard et à ses favoris. En 1397, Richard fit arrêter le duc de Gloucester et les earls de Warwick et d'Arundel. Ce dernier fut exécuté tandis que Warwick fut condamnée à l'emprisonnement à vie. Gloucester était déjà mort lors du procès. Cf. Saul, Richard II, op. cit., p. 366 et suivantes ; A. Goodman, The Loyal Conspiracy, The Lords Appellants under Richard II, Londres, 1971.

${ }^{39}$ Cf. Grady, "The Generation of 1399", dans The Letter of the Law. Legal Practice and Literary Production in Medieval England, éd. E. Steiner et C. Barrington, Ithaca et Londres, 2002, p. 202-229, p. 223-225.

${ }^{40}$ Mede est en moyen-anglais un terme extrêmement complexe dont les significations s'étendent de la récompense divine à la plus basse des corruptions, en passant par toutes les nuances de la rétribution. Langland joue largement de cette polysémie.
} 
Au passus IV enfin, Reson arrive à la cour, alors que Pees se plaint au roi des agissements de Wrong. Celui-ci est mis en procès, puis Reson prononce un discours contre Wrong et Mede. Le roi prend son parti et celui de Conscience et les prend comme conseillers.

La description du gouvernement idéal dans le prologue du poème, et plus généralement la conception de la royauté présente dans la première vision, a suscité de nombreux débats parmi les érudits ${ }^{41}$. Nous n'évoquerons là que la version B, écrite sans doute à la fin des années 1370 , c'est-à-dire à la fin du règne d'Édouard III, marquée par une crise liée à plusieurs éléments : des pertes importantes en France (c'est l'époque de la reconquête de Charles V), une crise économique... Le mécontentement s'est particulièrement exprimé au "Bon Parlement" de 1376, où les Communes (soutenus par certains lords) ont tenté de démettre certains membres du gouvernement royal et d'instaurer un programme réformateur, par la voix du premier Speaker de l'histoire, Peter de la Mare ${ }^{42}$. Concrètement, ce fut un échec et John of Gaunt, qui prit le gouvernement en main, réagit vigoureusement l'année suivante. Mais les conceptions réformatrices du "Bon Parlement" ont marqué les chroniqueurs et sans doute bien d'autres gens, dont Langland. Pourtant, sa présentation d'un parlement par le biais d'une fable, celle des rats, des souris et des chats, qui survient juste après la fondation idéale de la royauté, suscite un certain nombre d'interrogations. C'est un des éléments qui a parfois conduit à qualifier Langland d'un tenant de l'absolutisme. En voici le texte :

En un clin d'œil, une bande de rats et de petites souris arrivèrent en courant : ils étaient plus d'un millier à s'assembler pour discuter de leur intérêt commun ; il y avait en effet à la cour un chat, qui venait quand il en avait envie, qui se jetait sur eux, les attrapant aisément et jouant un jeu mortel, les faisant sauter d'une patte à l'autre.

"Par peur des périls qui nous environnent, c'est à peine si nous osons montrer le bout du museau ! Et si nous protestons contre ses petits jeux, il nous fera sentir son déplaisir. Il nous griffera, il nous étrillera, il nous serrera entre ses griffes, si bien que, lorsqu'il nous lâchera, la vie nous sera odieuse. Si seulement nous pouvions imaginer un plan pour résister à sa tyrannie, alors nous serions comme des lords et vivrions à notre aise".

Un rat de renom, à la langue éloquente, proposa une parfaite solution pour les sauver : "Dans la cité de Londres, dit-il, j'ai vu des gens portant autour du cou des colliers brillants et des cols habilement fabriqués; ils se promènent sans attache dans la garenne et les espaces ouverts, aussi loin qu'il leur plaît dans le monde, c'est comme je le dis. Il me semble, par le Christ, que si des cloches étaient attachées à leurs colliers, on pourrait savoir où ils vont, et s'enfuir. Et donc, dit le rat, le bon sens m'apprend qu'il faut acheter une cloche de cuivre ou d'argent brillant, l'attacher à un collier - c'est notre intérêt commun - et la pendre autour du cou du chat. Alors, nous saurons quand il bouge, quand il se repose, ou quand il prend son plaisir au-dehors; et s'il est d'humeur joueuse, nous le verrons bien, et nous pourrons nous montrer, et s'il est en colère, nous pourrons faire attention et nous écarter de son chemin".

Toute la foule des rats approuvait ce raisonnement ; mais lorsque la cloche fut achetée, et attachée au collier, il n'y eut pas dans toute la bande un rat qui osât - fût-ce pour tout le royaume de France ou toute l'Angleterre accrocher la cloche autour du cou du chat. Ils réalisèrent qu'ils n'étaient pas assez hardis, et que leur stratagème était un malheureux échec. Et vains furent tous leurs efforts et leur longue délibération.

Une souris qui avait, à mon avis, beaucoup de bon sens, sortit des rangs, se planta fermement devant la foule des rats et prononça ces mots : "Même si nous devions tuer le chat, un autre apparaîtrait à sa place, pour nous étriper tous, bien que nous nous soyons cachés sous les sièges. C'est pourquoi je conseille à toute la communauté de laisser le chat tranquille, et de ne pas être si téméraires que de lui montrer la cloche. Aussi longtemps qu'il est occupé à attraper des lapins et à se nourrir de venaison, il ne voudra pas de nos carcasses. Ne faisons rien qui porte atteinte à sa dignité. Mieux vaut un petit inconvénient qu'une catastrophe durable. Et même si nous nous débarrassions d'un ennemi particulier, le problème général ne serait pas résolu. J'entends encore mon père me dire, il y a sept ans: 'C'est une cour bien misérable que celle où le chat est un chaton'. Les Écritures, si vous vous donnez la peine de les lire, en témoignent : "Ve terre ubi puer rex est..." ${ }^{43}$. Car, voyez-vous, les rats deviendraient si actifs pendant la nuit, que personne ne pourrait fermer l'oeil ; nous, les souris, dévorerions le

\footnotetext{
${ }^{41}$ Pour une étude détaillée des conceptions du gouvernement chez Langland, nous renvoyons à notre ouvrage, Une Angleterre entre rêve et réalité, op. cit..

${ }^{42}$ Cf. Holmes, The Good Parliament, op. cit., passim.

43،Malheur au pays où le roi est un enfant..." (Eccl. 10: 16). Richard II n'avait que 10 ans quand il fut couronné roi en juillet 1377 .
} 
grain des hommes, pendant que les rats déchireraient leurs habits, si nous n'étions retenus par la peur que le chat de la cour ne fonde sur nous soudainement ; vous les rats, laissés à vous-mêmes, seriez bien incapables de vous gouverner.

En ce qui me concerne, dit la souris, je prévois de si graves conséquences, que je suis résolument contre toute provocation envers le chat ou le chaton, et que je suis opposée à toute discussion sur ce collier, qui ne m'a jamais rien coûté. Et même si j'avais contribué de mes deniers à son achat, je ne le dirais pas ! Je préfère supporter sa volonté et le laisser faire comme il lui plaît. Qu'ils aillent, tous les deux, librement, chasser où ils veulent. À tout homme raisonnable ici présent, mon avertissement est : occupez-vous de vos affaires !"

(Ce que ce rêve veut dire, bonnes gens, je préfère vous laisser l'interpréter. Dieu le sait, je n'oserais pas.)!

Piers Plowman B Prol., vers 146-210

Cet exemplum a soulevé de nombreuses questions, par rapport à ses sources, à ses références historiques précises, mais aussi à sa signification plus générale. Les premières sont maintenant bien cernées, même si nous ne connaissons pas la source précise de Langland ${ }^{44}$. Il a été utilisé à plusieurs reprises dans des sermons contemporains, par exemple dans celui que l'évêque de Rochester Thomas Brinton prononça, justement, durant le "Bon Parlement",45. Or, ce sermon avait des accents réformateurs et constituait un encouragement pour les Communes. Reste que l'usage qu'en fait Langland est ambigu. Selon Anna Baldwin, cet exemplum montrerait le peu de considération que Langland a pour les Communes ${ }^{46}$, mais une autre interprétation est donnée par Elisabeth Orsten. Cette dernière met en parallèle le discours du rat, qui propose de surveiller le chat, et celui de la souris, qui est contre cette proposition. De fait, c'est la raison qui guide le rat ainsi que l'intérêt commun (commune profit). Or, ces deux éléments sont très positifs dans le poème. L'intervention de la souris est en revanche hautement suspecte, tant dans sa construction que dans les termes employés, qui soulignent son exagération : nombre de mots expriment une grande violence (tuer, étriper, détruire...) et de manière plus générale, son discours reflète surtout la peur. Le vocabulaire utilisé par ces deux animaux s'oppose donc en bien des points : d'un côté, le rat en appelle à la raison et au bien commun, de l'autre, la souris joue sur la peur et sur la violence. La conclusion d'Elisabeth Orsten nous semble donc tout à fait plausible : "À la surface, donc, nous avons une fable dirigée contre les ambitions du 'Bon Parlement'. Mais un regard plus précis sur la souris révèle qu'il y a une attaque très légèrement voilée de John of Gaunt et de son Parlement de $1377^{\prime 47}$. La fable des rats, comme bien d'autres éléments dans le poème, suggère donc un Langland en faveur du "Bon Parlement" et de son esprit réformateur, surtout lorsque cette interprétation est mise en relation avec le fait que cette histoire a été utilisée par Brinton pour encourager les Communes. Il y a bien critique d'un parlement mais, comme dans Richard et dans Mum, cette critique porte d'abord sur le fait que ses membres ne remplissent pas leur fonction, celle de parler et d'instaurer un dialogue dans le sens du bien commun (le rat), et non dans celui de la violence qui conduit en fait à l'autocensure (la souris).

Chez Chaucer, la critique est à première vue de nature différente. The Parliament of Fowles n'est pas directement lié à une critique sociale et politique, même si beaucoup ont tenté des interprétations conjoncturelles du poème (la plus fréquente étant celle où le parlement des oiseaux ferait en fait référence au mariage de Richard II et d'Anne de Bohème).

\footnotetext{
${ }^{44}$ Cf. E. Orsten, “The Ambiguities in Langland's Rat Parliament”, Medieval Studies 23, 1961, p. 216-239. Pour un tableau de la présence fréquente de cette fable venant d'Orient dans les sources occidentales, voir P. F. Baum, "The Fable of belling the cat", dans Proverbia in Fabula, Essays on the Relationship of the Proverbs and the Fable, P. Carnes éd., Berne, 1988, p. 37-46.

${ }^{45}$ The Sermons of Thomas Brinton, Bishop of Rochester (1373-1389), éd. M.A. Devlin, 2 vol., Londres, 1954, vol. 2, p. 316-321. Pour les circonstances de ce sermon, voir Holmes, The Good Parliament, op. cit., p. 103-104.

${ }^{46}$ A. Baldwin, The Theme of Government in Piers Plowman, Cambridge, 1981.

${ }^{47}$ Orsten, "The Ambiguities", art. cité, p. 238.
} 
Ce n'est pas ce qui nous retiendra. Un des traits caractéristiques du parlement de Chaucer est que tout le monde y parle et que la cacophonie domine, malgré tous les efforts de la déesse Nature. C'est après l'intervention de chacun des trois aigles qui désirent la belle posée sur la main de Nature, que le désordre éclate :

Le bruit des oiseaux, pour que leur soit donné congé, retentit fortement: "Que cela soit fait, et laissez-nous aller !" Je pensais bien que le bois allait trembler. "Allez, crièrent-ils, hélas, vous allez nous détruire. Quand votre maudite dispute aura-t-elle une fin ? Comment un juge pourra-t-il croire une des partis pour le oui ou pour le non, sans aucune preuve?"

L'oie, le coucou, et aussi le canard, crièrent ainsi "kek kek! coucou ! quouac quouac !", si haut que le bruit transperça mes oreilles. L'oie dit: "tout cela ne vaut pas une mouche ! Mais je ne peux concevoir un remède Et je dirai mon verdict nettement et sans délais, pour les oiseaux d'eau, qu'ils soient contents ou en colère !”

"Et moi pour les oiseaux à vers, dit le coucou fou, je veux de ma propre autorité, pour le bien commun, reprendre maintenant l'accusation, car nous délivrer est grande charité". "Tu peux bien attendre un peu, pardi !, dit la tourterelle, si c'est notre volonté, un homme peut parler, mais il pourrait être aussi bien qu'il se taise.

Je suis un oiseau à grain, un des plus indignes, cela je le sais bien, et de peu de savoir. Mais il est mieux que la langue d'un homme se repose plutôt que d'interférer avec de tels faits, sur lesquels il ne peut conseiller ni chanter; et celui qui le fait est un oiseau qui s'accable bien lui-même, car un office non confié est souvent nuisible".

Parliament of Fowles, vers 491-518

En fait, toutes les catégories d'oiseaux autres que les oiseaux de proie s'insurgent de ce que ces derniers monopolisent la réunion avec leurs propres préoccupations, au détriment de tous. Et chacun de prendre la parole au nom de son groupe, non sans provoquer des animosités comme en témoigne la réponse de la tourterelle au coucou. Devant cette cacophonie, Nature décide de faire choisir un représentant par catégorie d'oiseaux. Au départ, du moins avec la classe la plus noble d'oiseaux, cela semble à peu près fonctionner : le faucon qui est élu fait un beau discours et sait se faire respecter. Mais par la suite, la cacophonie reprend, si bien que Nature décide finalement que le choix sera celui de la belle aigle, même si elle lui conseille de prendre celui de plus haute naissance. Ce n'est qu'avec cette intervention que l'ordre est rétabli. La critique semble donc avant tout porter sur le fait que tout le monde parle trop et sans autorité réelle alors que, comme le coucou, ils disent prendre la parole avec autorité.

Comme chez Langland, il nous semble que ce parlement des oiseaux (qui est aussi un parlement d'animaux) révèle avant tout la difficulté de parler ensemble, pour arriver à une décision qui n'aille ni contre l'autorité reconnue (Nature), ni contre le bien commun (en l'occurrence, le choix d'une compagne pour tous). Il suggère également, nous semble-t-il, la difficulté pour les membres "inférieurs" de la société politique de justifier leur part d'autorité, pourtant reconnue par la nomination de portes-paroles. Ces difficultés se reflètent d'ailleurs dans les controverses entre érudits sur l'interprétation de ce texte. Selon Paul Olson par exemple, qui a une vision très optimiste du poème, le débat sur l'amour est d'abord "un véhicule pour une discussion de la nature du social et de l'amour social en général" et le poème est une étude "sur la manière dont les gens agissant dans des corps constitués règlent les problèmes de loyauté, d'objectifs, de buts, de conflit ou d'intérêt et d'organisation" 48 . Mais Russel Peck a justement objecté que le parlement est en soi un échec politique ${ }^{49}$. Cela dit, le parlement se termine dans la concorde, car dans les derniers vers, les oiseaux chantent un rondelet d'amour et d'unité. Comme toujours en fait, Chaucer ne donne pas de réponse toute faite. En montrant les difficultés qu'ont les groupes d'oiseaux à parler ensemble, il

\footnotetext{
${ }^{48}$ Olson, "The Parliament of Fowles", op. cit., p. 53-54.

${ }^{49}$ R.A. Peck, "Love, Politics and Plot in the Parlement of Foules", Chaucer Review 24/4, 1990, p. 290-305, p. 293.
} 
suggère aussi la nécessité et la validité de cette discussion. Et Nature, qui représente l'autorité, prend bien soin d'écouter tout le monde, avant de prendre sa décision.

$* * * * * * *$

En fait, on le voit, que la critique porte sur le silence ou le bruit désordonné, les réflexions des poètes se rejoignent sur un certain nombre de points : dans tous les poèmes se pose la question de la validité du discours et de qui a autorité pour le faire, et cela en lien avec le conseil et plus généralement encore avec la question du bon gouvernement : le bien commun est une préoccupation partagée par tous les poètes, qui s'invitent ainsi dans le débat politique. Richard the Redeless et Mum and the Sothsegger, tout d'abord, s'interrogent de manière approfondie sur le conseil donné; le parlement, constitué autant des lords que des Communes, est pour eux un rouage essentiel et c'est pour cette raison que leurs critiques sont si vives. Cela confirme une évolution bien ancrée à la fin du XIV et au début du XV $\mathrm{XV}^{\mathrm{e}}$ siècles. Mais pour nos auteurs, il existe d'autres discours de conseil tout aussi valides. Dans leurs critiques se reflète en effet le point de vue de gens qui ne sont pas dans le premier cercle du roi, mais qui connaissent bien les rouages du gouvernement. Ce point de vue n'est donc pas neutre, et il est renforcé par le fait que, dans chaque poème, le narrateur insiste aussi sur l'importance de son propre conseil, même s'il doit pour cela faire des concessions. En effet, lorsqu'il n'est pas institutionnel, le conseil au prince est victime d'un paradoxe essentiel, comme l'a noté Judith Ferster à propos des miroirs au prince, textes de conseil par excellence :

“D'un côté, l'accord est large sur le fait que les miroirs aux princes adoptent le point de vue du gouvernant parce que l'auteur est subordonné à et aspire à la faveur du prince. En conséquence, il fait luimême preuve d'humilité, flatte le gouvernant et promeut de manière appropriée une vue hiérarchique de la société dans laquelle les gouvernants exercent le pouvoir et les sujets obéissent. Mais de l'autre côté, la prémisse contraire est également et plus subversivement fondamentale pour le genre : pour bien régner, le roi doit être dirigé"

L'auteur de Richard évite le paradoxe parce qu'il s'adresse fictivement à un roi qui n'est plus de ce monde. Dans Mum, il faut en revanche la quête entière du narrateur et la bénédiction de l'autorité du poème, l'apiculteur (qui est un laïc et non un clerc), pour qu'il se décide à affirmer la pertinence de son conseil. La résolution du paradoxe se fait donc au prix d'un compromis important, le positionnement en marge des centres du pouvoir ${ }^{51}$. Et l'échec des représentants en parlement à remplir leur tâche - qu'il soit volontaire ou non - constitue une justification supplémentaire pour le poète. Ainsi le topos du conseil fait-il partie d'un projet politique concret, celui d'empêcher que ne se reproduisent les événements dramatiques qui ont abouti à la déposition du roi, en même temps qu'il permet de replacer ces événements dans une réflexion plus vaste. Pour les poètes, le conseil ne peut être le seul apanage des membres des instances institutionnelles, même si celui-ci est indispensable. L'enjeu est trop important. Ces deux poèmes révèlent donc une perception relativement claire et tranchée de la place du parlement au sein de la construction du dialogue politique anglais : essentielle, mais pas unique. D'autres voix, dont celles des auteurs, doivent se faire entendre.

\footnotetext{
${ }^{50}$ Ferster, Fictions of Advice, op. cit., p. 40. Sur les miroirs au prince, voir J.-P. Genet, "La formation du genre des miroirs des princes en Occident", dans Religion et mentalités au Moyen Âge, Mélanges en l'honneur d'Hervé Martin, éd. S. Cassagnes-Brouquet, A. Chavou, D. Pichot et L. Rousselot, Rennes, 2003, p. 531-541.

${ }^{51}$ Cf. H. Barr et K. Ward-Perkins, "'Spekyng for one's sustenance' : the Rhetoric of Counsel in Mum and the Sothsegger, Skelton's Bowge of Court, and Elyot's Pasquil the Playne », dans The Long Fifteenth Century. Essays for Douglas Gray, éd. H. Cooper et S. Mapstone, Oxford, 1997, p. 249-72, p. 256.
} 
Langland et Chaucer ont des positions plus subtiles et n'ont pas de réponse tranchée. Leur réflexion sur le parlement doit en fait être replacée dans le cadre plus général de leur réflexion constante sur le fait qu'il n'existe pas un seul discours mais plusieurs, la question étant de savoir lesquels sont valides. Et chez ces deux auteurs, dont la poésie est pourtant si différente (formellement en tout cas), les thèmes de la polyphonie du discours et de la revendication d'un partage de l'autorité sont essentiels. Paul Strohm, dans son ouvrage Social Chaucer, l'a fort bien résumé pour Chaucer, en suggérant que la construction des Canterbury Tales, dans lesquels chaque conte est narré par un personnage différent, aussi bien du point de vue psychologique que social, constituait une réponse littéraire aux transformations des rapports sociaux à la fin du Moyen Âge :

\begin{abstract}
"Les variations génériques et stylistiques de Chaucer, et sa multiplication des différentes vocalités par lesquelles cette variation est soutenue, peuvent être vue comme une réponse de médiation au factionnalisme et à la contradiction au sein de sa propre expérience sociale... L'entreprise esthétique de Chaucer de définition d'un espace littéraire permettant une libre interaction de différentes formes et styles peut être placée dans une relation réciproque avec l'entreprise sociale de définition d'un espace public hospitalier pour les différentes classes sociales aux envies sociales différentes" ${ }^{, 52}$.
\end{abstract}

The Parliament of Fowles, qui a été écrit avant les Canterbury Tales, constitue également une réponse à ces transformations. En faisant parler les différents groupes d'oiseaux, en insistant sur les différentes tentatives de médiation de Nature (même si c'est elle qui a l'autorité finale), Chaucer reconnaît et accepte l'importance de ces transformations. En ce qui concerne Langland, nous avons tenté de montrer ailleurs que, malgré des différences, cette réflexion était également présente chez ce dernier ${ }^{53}$.

Tous les poèmes que nous avons évoqué (Richard et Mum y compris) s'interrogent, bien que de manière différente, sur ces transformations et sur leurs liens avec les notions fondamentales de discours, de conseil, d'autorité et de bien commun. Ces thèmes sont présents dans tous les domaines envisagés par leurs auteurs. La place qu'ils accordent au parlement, que ce soit par le biais de la fable, de la narration ou de la satire, renforce cette approche. Elle est significative, autant pour la reconnaissance de ces transformations que pour le rôle du parlement comme catalyseur de ces dernières et comme lieu d'ouverture de la société politique anglaise à la fin du $\mathrm{XIV}^{\mathrm{e}}$ siècle. Cependant, le parlement n'est pas le seul lieu de l'expression d'un dialogue en perpétuelle construction : nos poètes ont l'ambition d'y participer tout autant et se sentent en droit de le faire, même s'ils se situent en général dans une position marginale (comme l'auteur de Mum) ou au moins de prise de distance (c'est le cas de Chaucer par exemple). En cela, ils ne sont pas isolés, car la poésie anglaise a été, à la fin $\mathrm{du} X \mathrm{XV}^{\mathrm{e}}$ siècle et au début du $\mathrm{XV}^{\mathrm{e}}$, un médium essentiel de réflexion sur la société anglaise et ses évolutions.

CNRS - Centre d'études supérieures de civilisation médiévale (Poitiers)

\footnotetext{
${ }^{52}$ P. Strohm, Social Chaucer, Cambridge et Londres, 1989, p. 163-164.

${ }^{53}$ Une Angleterre entre rêve et réalité, op. cit..
} 